\title{
Design and Implementation of ICT-Based System for Information Management of Livestock Farm
}

\author{
Hyungi Kim ${ }^{1}$, Seokkyun Jeong ${ }^{2}$ and Hyun Yoe ${ }^{3 *}$ \\ 1,2,3 Dept. Of Information and Communication Engineering, Sunchon National \\ University, Suncheon, Jeollanam-do, Republic of Korea \\ \{kimhyungi,sk_jeong, yhyun\}@sunchon.ac.kr
}

\begin{abstract}
This paper proposes ICT based system for information management of livestock farm to provide efficiency operation in livestock farm by managing information of livestock farm such as livestock information and environment information and fire infôrmation Proposed system provides optimal breeding environment by monitoring real-time information of livestock farm and manage overall information of livestock such as disease forecasting and estrus detection and delivery time. It is expected to increase productivity and earnings rate in livestock farm by systematically managing livestock information and economically operating livestock farm.
\end{abstract}

Keywords: WSN, ICT, Middle ware, Livesfock, Livestock Farm

\section{Introduction}

Information and communications technolog (ICT) is often used as an extended synonym for information technology, but is a more specific term that stresses the role of unified communications and the integration of tecommunications (telephone lines and wireless signals, computers as well as hecessary enterprise software, middleware, storage, and audiovisual systems, which enable users to access, store, transmit, and manipulate information [1, 2].

The Information and communications technology has been applied through a variety of fields including defense, mediclne, road transport, security and realizes advancement of the living standard. So the Information and communications technology has applied to livestock industry for increasing productivity [3, 4].

The current domestic livestock industry competes with advanced countries of livestock industry because of inevitable circumstance such as FTA agreement and increase cost of feed. livestock farms have received significant damages from contagious livestock diseases such as foot-and-nouth disease and AI, which lead to increased mortality rate and financial damage of livestock farms[5].

in order to solve such problems, livestock farms make optimal breeding environment using systematically scientific technology and need integration system of livestock farm to increase productivity by reducing produce cost and mortality rate. However, the existing developed systems lake integration technology about information of livestock farms [6].

Accordingly, This Paper proposes ICT based system for information management of livestock farm by managing information of livestock farm such as livestock information and environment information and fire information. Proposed system provides optimal breeding environment by collecting real-time environment information installed environment sensor in

${ }^{*}$ Corresponding Author 
livestock farm and monitoring service to detect fire information. In addition, It generally manages livestock information such as estrus detection and delivery time by collecting activity information of livestock.

Therefore, it is expected to reduce labor force and systematically operate livestock farm.

The composition of this paper is as follows. Chapter 2 explains design of ICT based system for information management of livestock farm. Chapter 3 explains implementation of proposed system. Lastly, Chapter 4 will conclude this paper by describing conclusion.

\section{Design of Proposed System}

This paper proposes system that livestock farms could be economically operated by making disease forecasting and estrus detection and delivery time based on information of livestock activity. Also, Optimal breeding environment of livestock could be provided by monitoring and collecting environment information in livestock farm. The fire detection sensors are deployed such as spark detection sensor, smoke sensor, temperature sensor in livestock farm. Fire detection server stores collected fire information in database and provides fire detection service to user in real time.

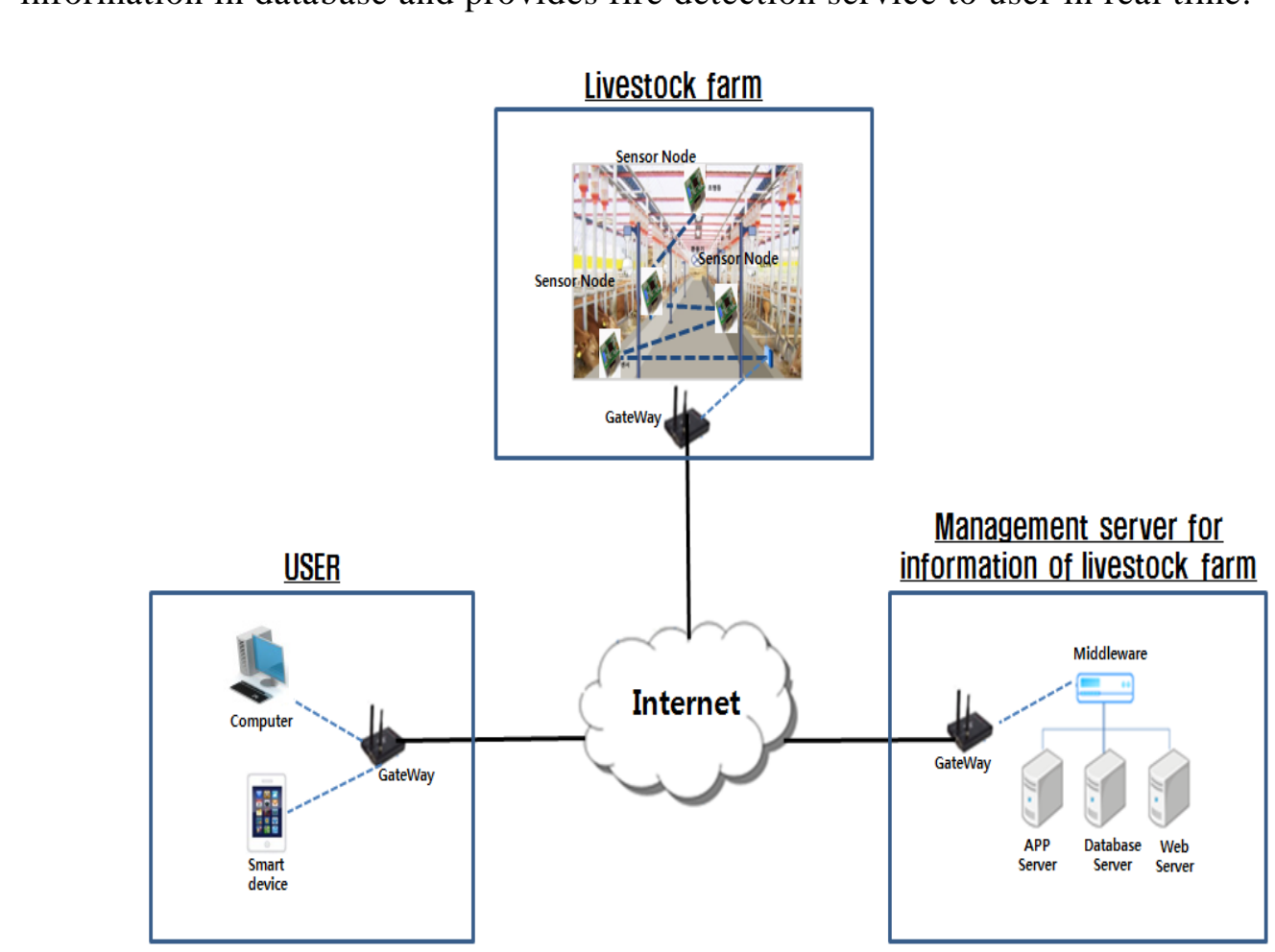

Figure 1. The Structure of Proposed System

Figure 1 is structure of ICT based system for information management of livestock farm. It consists of livestock farm, management server for information of livestock farm and User. 


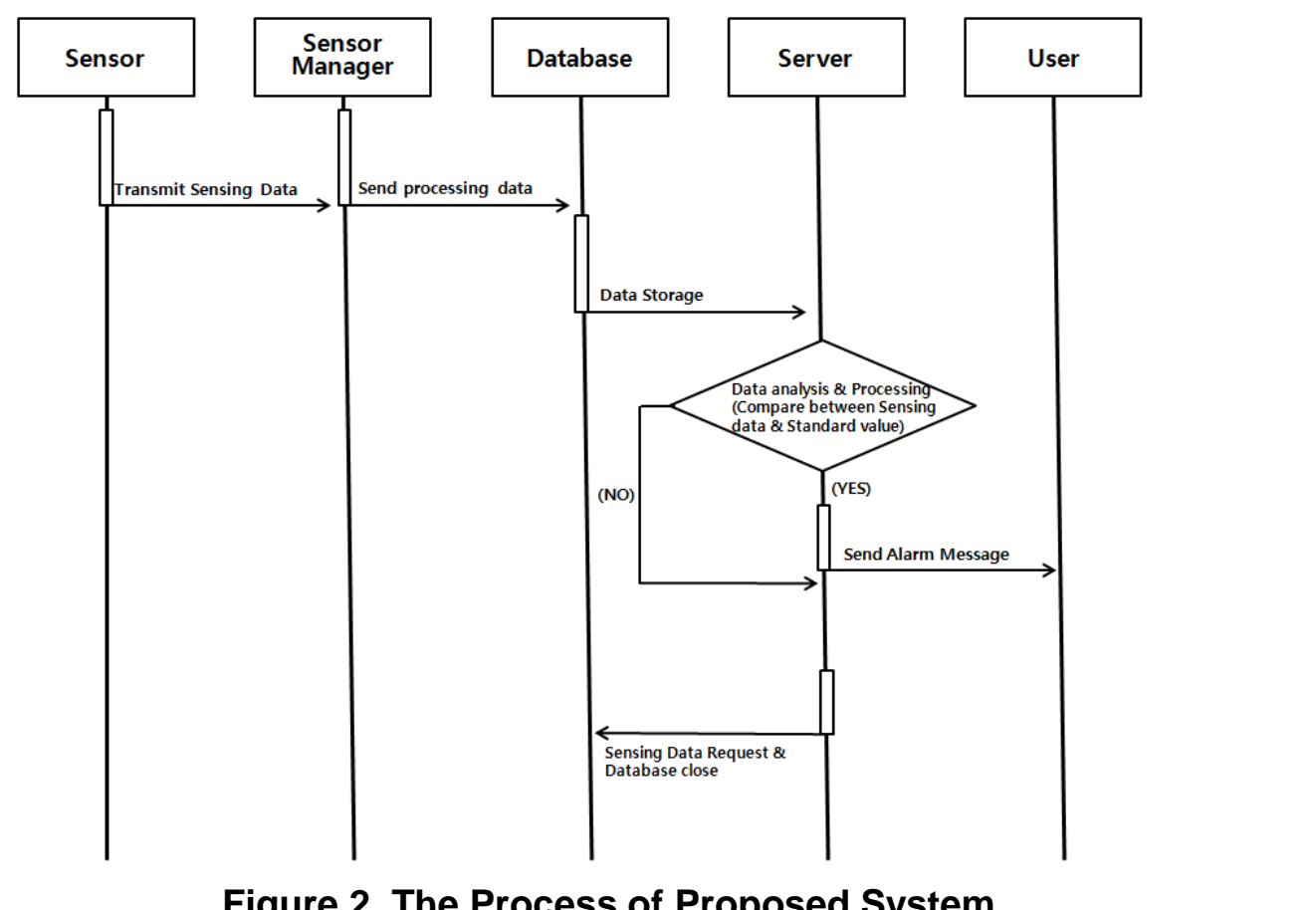

Figure 2. The Process of Proposed System

Figure 2 is process of proposed ICT based system for information management of livestock farm. This process consists of fire environment sensor node, sensor manager, database, management server for information of hivestock farm and user. Sensor node for collecting information of livestock larm like fire detection and livestock information transmits collected information to sensor manager. A sensor manager formats information collected from sensor node so as to save in database and converts units suiting to measuring factors and saves processec data in database using update query. Management server for information of livestock farm is updated from database in real time and provides alarm service like disease forecasting, estrus detection and delivery time to user.

\section{Implementation of Proposed System}

Figure 3 is The Middle ware for information management of livestock farm. Alphabet A is section of environment information such as temperature, humidity, illumination in livestock farm. Alphabet B is section of spark detection, smoke data in livestock farm. Through establishment of test bed of ICT based system for information management of livestock farm, the study confirmed that data collected from a variety of sensor node was normally stored in database hrough middleware without malfunction. If fire detection device detect fire, fire alarm is worked well. 


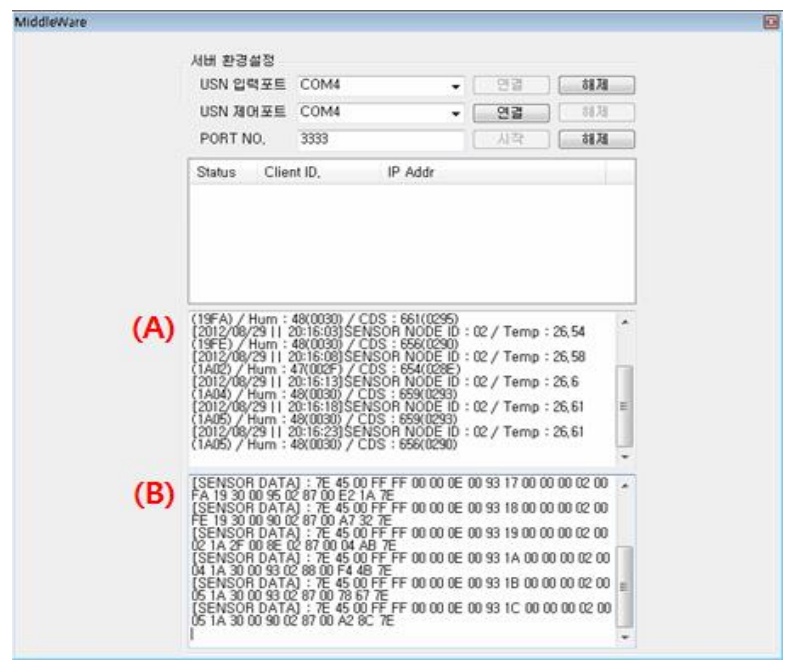

Figure 3. The Middle Ware for Proposed ICT bầsed System

Figure 4 is The System for information management of livestock farm. Environment setting of system consists of input port, control port and port number to connect middle ware. Alphabet A and B is section of monitoring real time livestock farm to detect novel anomaly. Alphabet $\mathrm{C}$ and $\mathrm{D}$ is section of collecting environment iniormation like temperature, humidity, illumination, wind and fire information such spark, smoke to manage efficient livestock farm.

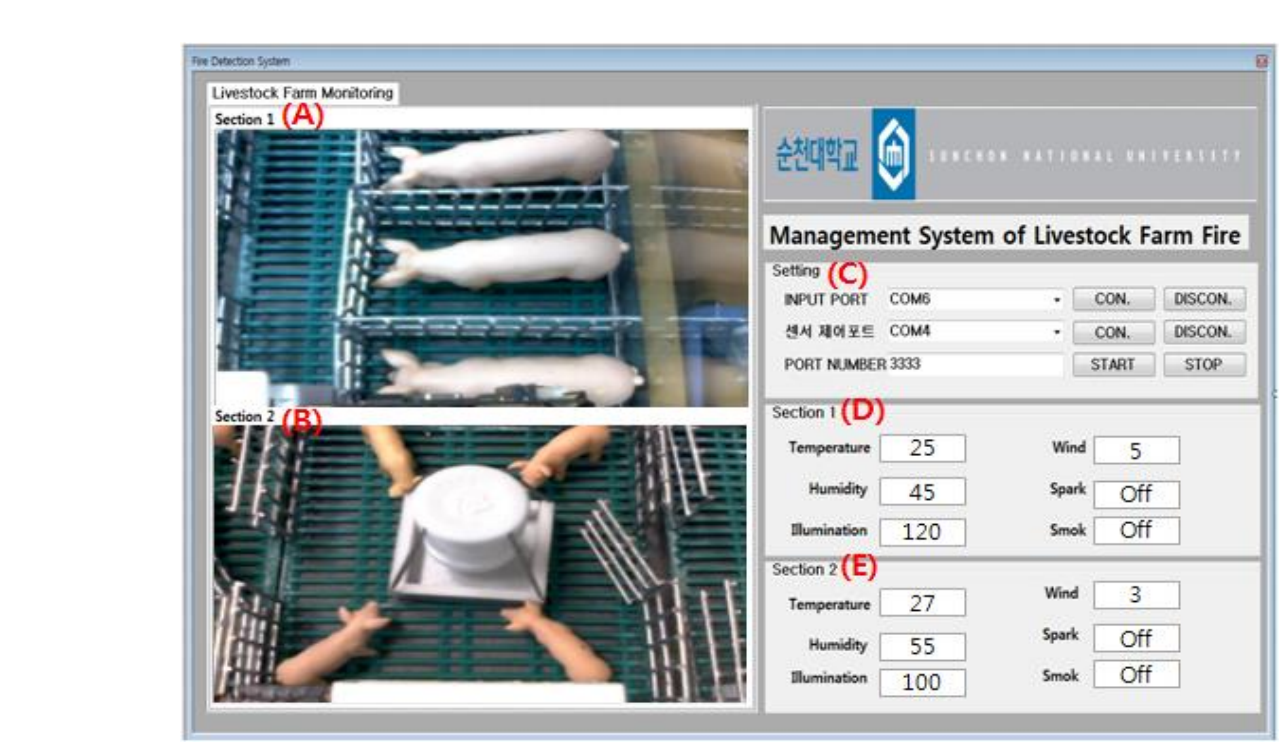

Figure 4. The System GUI for Proposed System

\section{Conclusion}

Livestock farms reduce cost of production for increasing rate of return to investment and need to operate efficient livestock farm by using ICT based system.

This paper proposes ICT based system for information management of livestock farm to provide methodical and efficiency operation in livestock farm. Proposed system aim to provide a variety of alarm service such as livestock information, environment 
information and fire information by storing collected data from livestock farm in database using the wireless sensor network.

It is also expected that this system could efficiently manage livestock farm and avoid economic losses by rapidly detecting livestock farm fire.

\section{Acknowledgements}

"This research was financially supported by the Ministry of Education (MOE) and National Research Foundation of Korea(NRF) through the Human Resource Training Project for Regional Innovation (No.2011-0202 )."

"This research was supported by the MSIP(Ministry of Science, ICT and Future Planning), Korea, under the CITRC(Convergence Information Technology Research Center) support program (NIPA-2013-H0401-13-2008) supervised by the NIPA(National IT Industry Promotion Agency)."

\section{References}

[1] J.-H. Koo, T.-W. Jeong, S.-R. Lee and J.-H. Chom, "A Study on the Application of an Mntegrated Livestock Manure Management System Using u-ICT Focusing on liquid fertilization”, Journal of livestock housing and environment, vol. 18, (2012), pp. 1226-0274, pp. 7-12.

[2] J. H. Hwang, C. S. Shin and H. Yoe, "Study on an Agricultural Environment Monitoring Server System using Wireless Sensor Networks", Sensors, vol. 10, (2010), pp. 11189-11211.

[3] J. H. Hwang and H. Yoe, "A Study on the Context-A ware Middleware for Implementing Intelligent Service in Ubiquitous Livestock Barn based on Wireless Sensor Networks", Sensors, (2011), pp. 4539-4561.

[4] M. Yoon and J.-W. Chang, "Design and Implementation of an Advanced Cattle Shed Management System using a Infrared Wireless Sensor node and Suryeillance Camera", Digital Contents Society, vol. 12, no. 10, (2012), pp. 22-34.

[5] H. Jeong, C.-J. Yang and H. Yoe, "Study on the Livestock Activity Monitoring System Using Acceleration", Green and Smart Technology with Sensor Applications Communications in Computer and Information Science, vol. 338, (2012), pp. 297-302.

[6] J. H. Hwang and H. Yoe, "Sttdy of the Ubiquitous Hog Farm System Using Wireless Sensor Networks for Environmental Monitoring and Facilities Control", Sensors, (2010), pp. 10752-1077.

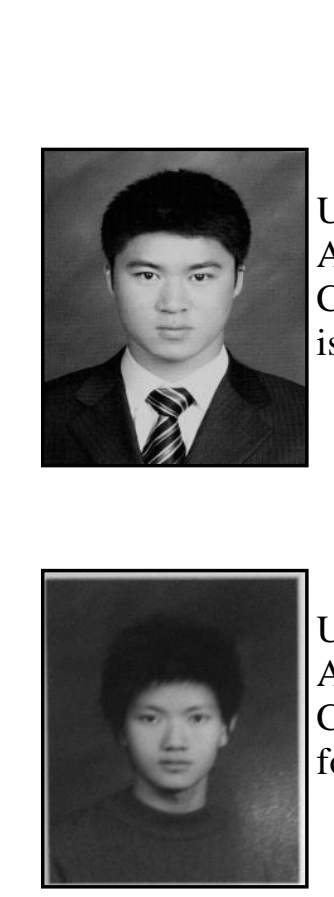

Hyungi Kim, is a graduate school student of Sunchon National University. Hyungi Kim is also a researcher of u-agriculture IT Application Research Center and Agriculture IT Convergence Support Center at Sunchon National University too. Hyungi Kim's research focus is Wireless Sensor Networks, MAC Protocol.

Seokkyun Jeong, is a graduate school student of Sunchon National University. Seokkyun Jeong is also a researcher of u-agriculture IT Application Research Center and Agriculture IT Convergence Support Center at Sunchon National University too. Seokkyun Jeong's research focus is Wireless Sensor Networks and Cloud Computing. 


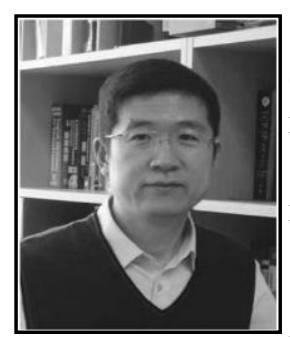

Hyun Yoe, is a professor in the department of Information and communication at Sunchon National University, South Korea. He received his M.S and Ph.D degree in Electronic Engineering from Soongsil University in 1987 and 1992, respectively. He has been a research staff in KT research Institute in 1987-1993 and ETRI in 1995 in Korea. He also has been researched at Georgia Institute of Technology in 1997-1998 in U.S.A. He is a member of IEEE, KICS and KSII. Dr. Yoe's research focus is sensor networks and ubiquitous applications for agriculture. He has investigated issues in ubiquitous sensor networks and wireless networks. He has applied sensor networks to ubiquitous agriculture. $\mathrm{He}$ is in charge of uARC(ubiquitous Agriculture IT Application Research Center) and AITCSC(Agriculture IT Convergence Support Center) which is supported by MKE(Ministry of Knowledge Economy), Korea.

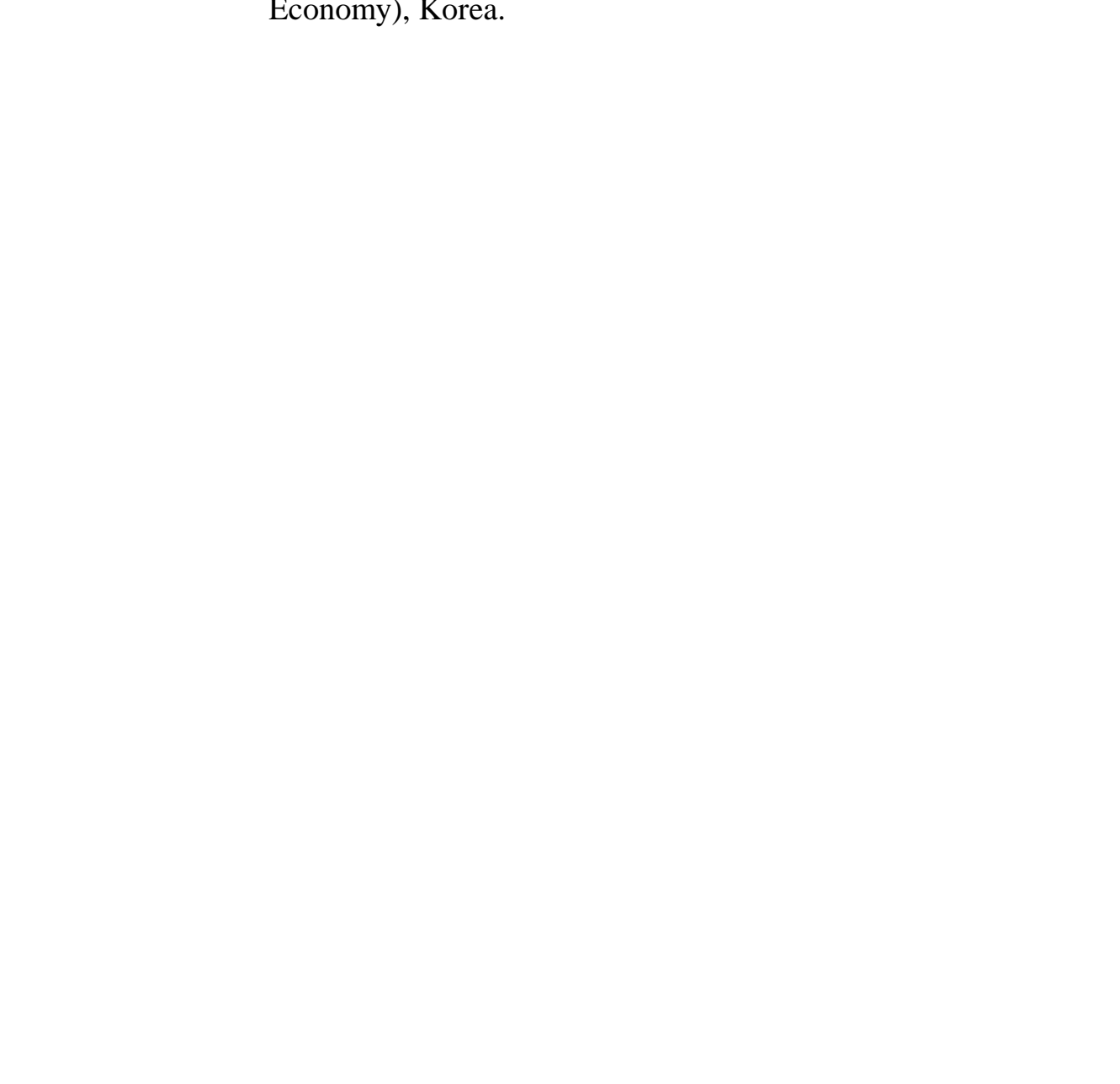

